\title{
Association between Patient's Basic Characteristic and Clark Level in Cutaneous Malignant Melanoma
}

\author{
Egie Rahmalaelani, ${ }^{1}$ Hermin Aminah Usman, ${ }^{2}$ Jono Hadi Agusni ${ }^{3}$ \\ ${ }^{1}$ Faculty of Medicine Universitas Padjadjaran, ${ }^{2}$ Department of Anatomical Pathology Faculty of \\ Medicine Universitas Padjadjaran/Dr. Hasan Sadikin General Hospital Bandung, ${ }^{3}$ Department of \\ Dermato-Venereology Faculty of Medicine Universitas Padjadjaran/Dr. Hasan Sadikin General \\ Hospital Bandung
}

\section{Abstract}

Background: Malignant melanoma is a cancer that begins in the melanocytes. Melanoma is commonly happened on older people, male and located on sun exposure body area, such as face, neck and lower limb. Melanoma cases occur less than 5\% of skin cancer but cause a majority of skin cancer deaths because melanoma has an aggressive behavior. Age, sex, location of lesion, and type of melanoma influences the melanoma invasion. The cutaneous melanoma invasion could be assessed with the Clark level. This study is aimed to determine the association between patient's basic characteristic and Clark level in cutaneous malignant melanoma.

Methods: This study used cross sectional analytic design. The medical records and histopathology slide from the Department of Anatomic Pathology Dr. Hasan Sadikin General Hospital from 2008-2012 took as a sample. Forty one data were meet the inclusion criteria. Furthermore, those data were analyzed using chisquare test.

Result: Overall, patient's characteristic in this study, commonly occurred on female (66\%), 50-59 years group age (35\%), non-cephalic/acral location (71\%), and the level V in Clark level (73\%). Location of lesion showed an association with Clark level $(\mathrm{p}=0.023)$, whereas, age and sex was not significant $(\mathrm{p}=0.679$ and $\mathrm{p}=0.389$ ).

Conclusions: There is an association between location of lesion and Clark level [AMJ.2017;4(1):25-9]

Keywords: Basic characteristic, cutaneous melanoma, Clark level, malignant melanoma

\section{Introduction}

Malignant melanoma is a cancer that begins in the melanocytes, which gives tanned skin. ${ }^{1}$ Although primary site of melanoma is skin, but it could occurs on the other part of the body such as eyes, mouth and genital. ${ }^{1}$ Melanoma is commonly happen on older people, but nowadays it is also could occured on younger people. This condition is due to aging condition which reduces skin immunity. ${ }^{2}$ Ultraviolet rays are the main risk factors for malignant melanoma. Fair-skinned people had high vulnerability to malignant melanoma, which is 20 -fold compared to the dark-skinned people and the most common locations of malignant melanoma are the face, neck, and lower limbs. However, the location of malignant melanoma on dark-skinned people is not on the area that exposed by the sunlight, but the protected areas such as the soles, palms, and nails bed., ${ }^{1,3}$ In United State, male had a higher rate of melanoma occurrence than female. ${ }^{1}$ This was due to the most male's jobs that were exposed by the sun rays which could stimulated melanocytes to grow continuously.

Malignant melanoma rarely occurs compared to basal cell carcinoma and squamous cell carcinoma, but has higher aggressiveness, with its ability to spread to other organs or metastases. ${ }^{1}$ Metastases of malignant melanoma was more common through the lymphatic system and make it as one of the most deadly cancers with threefold mortality rate compared to the other skin cancers. ${ }^{5}$ Depth of invasion is an important

Correspondence: Egie Rahmalaelani, Faculty of Medicine, Universitas Padjadjaran, Jalan Raya Bandung-Sumedang Km.21, Jatinangor, Sumedang, Indonesia, Phone: +62 85720010023 Email: egee_rahma@yahoo.com 
prognostic factor in the evaluation of malignant melanoma. ${ }^{6}$ Patients of melanoma with superficial invasion have higher survival rate than patients with deeper invasion. ${ }^{6}$ Clark level is used to assess the depth of invasion of malignant melanoma on the skin. On level I, the depth of melanoma is still limited in the epidermal layer also called melanoma in situ. Level II, depth reaches a little layer of papillary dermis; level III, reaches across the papillary layer of the dermis; level IV, reaches the circular layer of the dermis; and level V reaches the subcutaneous layer. ${ }^{6}$ Therefore Clark level becomes important in determining the prognosis of malignant melanoma.

Homsi J et al. ${ }^{7}$ and Filamer et al. ${ }^{8}$ mentioned that, age, sex, location of lesion and type of malignant melanoma influence melanoma invasion. Thus, according to those data, the purpose of this study was to determine the association between patient's characteristic and Clark level in cutaneous malignant melanoma.

\section{Methods}

This study used cross-sectional analytic design, and took samples with total sampling method because malignant melanoma case rarely occurred. The study used secondary data, which are medical records and histopathology slides for assessing the Clark level. The medical records of patients who were diagnosed malignant melanoma from January 2008-December 2012 in Department of Anatomical Pathology Dr. Hasan Sadikin General Hospital Bandung, were taken for samples. The inclusion criteria in this study were the patients who were diagnosed cutaneous malignant melanoma complete with histopathology slides or paraffin blocks. The exclusion criteria were cutaneous malignant melanoma patients that were not complete either their data or histopathology slide or paraffin blocks. Clark level assessment is conducted by using a light microscope. On level I, the depth of melanoma is still limited in the epidermal layer also called melanoma in situ. Level II, depth reaches a little layer of papillary dermis; level III, reaches across the papillary layer of the dermis; level IV, reaches the circular layer of the dermis; and level V reaches the subcutaneous layer. ${ }^{6}$ The data were analyzed using chi-square test, and the hypothesis will be accepted if $\mathrm{p}<0.05$.

This whole research process had to get permission from the Health Research Ethics Committee of the Faculty of Medicine
Universitas Padjadjaran and Dr. Hasan Sadikin General Hospital Bandung

\section{Results}

There were 94 cases of malignant melanoma that recorded in Dr. Hasan Sadikin General Hospital in 2008-2012. Based on inclusion criteria, only 41 data can be analyzed. There were $14(35 \%)$ cases occurred in 50-59 group age with mean age 60.7 years old, and the most occured at female group (66\%). The most common location of malignant melanoma was non-cephalic/acral with $29(71 \%)$ cases, and 6 $(15 \%)$ cases occurred in other locations, such as the back, flank area, rectum, and thorax. Thirty $(73 \%)$ cases categorized into the level $\mathrm{V}$, which is the level that had been reached subcutaneous melanoma and no one at level I (Table 1).

The association between patient's characteristic and Clark level in cutaneous malignant melanoma which analyzed using chi-square test, showed that, only the location of lesion that had significant $(\mathrm{p}=0.023$ ) towards Clark level, whereas age and sex showed no significant results $(\mathrm{p}=0.679$ and $\mathrm{p}=0.389)$.

\section{Discussion}

Although malignant melanoma is commonly happened on older people but it also can be occurred on the younger people. ${ }^{1}$ In this study, the mean age is 60.7 years old and the oldest is 90 years old. This indicates that there might be genetic and immunity influences occurrence of malignant melanoma. Genetic influences can be caused by mutations in the gene CDKN2A (cycling-dependent kinase inhibitor) and B-RAF gene (part of cell signaling and growth). ${ }^{1}$ Whereas immunity influence caused by the immaturity of the children immunity and immunity decreasing while aging, which influence the skin ability to regenerate effectively. ${ }^{2}$ Age is one of prognosis factor in malignant melanoma and invasion of Clark level often used as other prognostic factors. ${ }^{3}$ In this study, the association between age and Clark level showed non significant $(p=0.679)$. This is similar with Anger et al. ${ }^{9}$ who mentioned that age does not affect the level of malignant melanoma invasion.

More than half of malignant melanoma cases in this study occurred in female, it is similar with Anger et al. ${ }^{9}$ in Brazil. In the study by Markovic et al. ${ }^{4}$ it is noted that the increasing of malignant melanoma cases in women 
Table 1 Respondents Characteristic

\begin{tabular}{|c|c|c|}
\hline Characteristic & $\mathbf{n}$ & $\%$ \\
\hline \multicolumn{3}{|l|}{ Age (years old) } \\
\hline $0-9$ & 0 & 0 \\
\hline $10-19$ & 0 & 0 \\
\hline $20-29$ & 1 & 2 \\
\hline $30-39$ & 1 & 2 \\
\hline $40-49$ & 4 & 10 \\
\hline $50-59$ & 14 & 35 \\
\hline $60-69$ & 11 & 27 \\
\hline $70-79$ & 7 & 17 \\
\hline $80-89$ & 2 & 5 \\
\hline $90-99$ & 1 & 2 \\
\hline \multicolumn{3}{|l|}{ Sex } \\
\hline Male & 14 & 34 \\
\hline Female & 27 & 66 \\
\hline \multicolumn{3}{|l|}{ Location of lesion } \\
\hline Cephalic* & 6 & 15 \\
\hline Non-cephalic/acral** & 29 & 70 \\
\hline Others \# & 6 & 15 \\
\hline \multicolumn{3}{|l|}{ Clark Level } \\
\hline I & 0 & 0 \\
\hline II & 1 & 2 \\
\hline III & 2 & 5 \\
\hline IV & 8 & 20 \\
\hline V & 30 & 73 \\
\hline Total & 41 & 100 \\
\hline
\end{tabular}

Note: *head, face, and neck, **hand, leg, sole, palm, \#back, flank area, rectum, and thorax

occurred due to the use of oral contraception (estrogen and progesterone) that can stimulate the proliferation of melanocytes and as well as the lifestyle changes, such as how to dress up daily. However, in these studies, most common of malignant melanoma occurred in male. This was due to the male's jobs that were exposed to the sun rays which could stimulated melanocytes to grow continuously. The prognosis of malignant melanoma in women is better than men, but actually sex is not the main factor affecting the prognosis of the patients, thus it is necessary to consider the other prognosis factors. ${ }^{3}$ In this study, the association between sex with Clark level showed no significant results $(\mathrm{p}=0.389)$ which similar with Anger et al. ${ }^{9}$
In this study, the location of non-cephalic/ acral had higher numbers than the cephalic. Study in Malaysia, Mexico, and Brazil (by Pailoor et al. ${ }^{10}$ Karam-Orantes et al. ${ }^{11}$ and Moris Anger et al. ${ }^{9}$ ) showed that the most common malignant melanoma is found in the extremities or the acral area. Race is one of a risk factors of acral malignant melanoma. ${ }^{12}$ Melanoma from sun-exposed skin showed frequent mutations in either B-RAF or NRAS gene (part of cell signaling and growth). In contrast, melanoma risen from sun-protected area showed a higher degree of chromosomal aberrations, the most common occurred in chromosome 11q13 which contains the cyclin D1 gene. Cyclin D1 positively regulates the activity of cycling dependent kinase, 
Table 2 Association between Respondents Characteristic with Clark Level

\begin{tabular}{|c|c|c|c|c|c|c|c|}
\hline \multirow{2}{*}{\multicolumn{2}{|c|}{ Characteristic }} & \multicolumn{5}{|c|}{ Clark level } & \multirow{2}{*}{ p } \\
\hline & & I & II & III & IV & V & \\
\hline \multirow[t]{10}{*}{ Age (years old) } & $0-9$ & 0 & 0 & 0 & 0 & 0 & \multirow{10}{*}{0.679} \\
\hline & $10-19$ & 0 & 0 & 0 & 0 & 0 & \\
\hline & $20-29$ & 0 & 0 & 0 & 1 & 0 & \\
\hline & $30-39$ & 0 & 0 & 0 & 0 & 1 & \\
\hline & $40-49$ & 0 & 0 & 0 & 0 & 4 & \\
\hline & $50-59$ & 0 & 0 & 1 & 3 & 10 & \\
\hline & $60-69$ & 0 & 1 & 0 & 0 & 10 & \\
\hline & $70-79$ & 0 & 0 & 1 & 3 & 3 & \\
\hline & $80-89$ & 0 & 0 & 0 & 1 & 1 & \\
\hline & $90-99$ & 0 & 0 & 0 & 0 & 1 & \\
\hline \multirow[t]{2}{*}{ Sex } & Male & 0 & 0 & 0 & 3 & 10 & \multirow{2}{*}{0.389} \\
\hline & Female & 0 & 0 & 2 & 5 & 20 & \\
\hline \multirow[t]{3}{*}{ Location } & Cephalic & 0 & 0 & 2 & 1 & 3 & \multirow{3}{*}{$0.023 *$} \\
\hline & Non-cephalic/acral & 0 & 1 & 0 & 7 & 21 & \\
\hline & Others & 0 & 0 & 0 & 0 & 6 & \\
\hline
\end{tabular}

Note: *p value significant $(\mathrm{p}<0.05)$

leading to phosphorylation of retinoblastoma protein promoting entry into mitosis, and acts as an oncogene. ${ }^{13}$ In fair-skinned people or the Caucasian, most location of malignant melanoma occurrences were at the face, neck, and lower limbs, however, in dark-skinned people or non-Caucasian, the location of the occurrences were exactly not the same, its occurred on the protected area such as the palm of legs, palm of hands, and nails bed.1,3 Melanoma was strongly associated with penetrative injury of the feet or hands. Local injury may promote acral melanoma through stimulation of melanocytic cell by fibroblast growth factor abundant in healing wounds. ${ }^{12}$

In this study, the significant result showed $(p=0.023)$ on the association between the location of the lesion and Clark level. This result was similar with Anger et al. ${ }^{9}$ Noncephalic/acral locations such as the palms and soles that have thicker skin than other parts of the body skin, they should have a slower level of invasion. Acral melanoma becomes invasive when the melanoma cells cross the basement membrane of the epidermis and malignant cells enter the dermis. Hyun Sun Park et al. ${ }^{14}$ mentioned that acral melanoma has radial growth. Histological characterized by proliferation of atypical melanocytes along the basal layer and associated with elongation of rete ridges. ${ }^{15}$

Although malignant melanoma has an aggressive behaviour, early stage treatment probably have fairly good prognosis. In this study, 30 cases were categorized as level V, which was the melanoma that has reached the subcutaneous layer. This result may occurred due to the lack of concerns for the patient's lesions in acral areas. This condition led to delays diagnosis of malignant melanoma which made the patients came to the doctor in the severe stage.

In this study, there were some limitations, including a limited time, the incomplete data, and Clark level assessment performed by medical students. However, it can be anticipated by prior training and supervision from the doctor.

In this study, it can be concluded that there is association between patient's characteristic and Clark level. Age and sex were not associated to Clark level. However, the location of the lesion has a significant association with Clark level.

References 
1. American Cancer Society. Melanoma skin cancer. 2012. p. 1-11. [Cited 20139 February]. Available from: https://www. cancer.org.

2. Yaar M, Gilchrest BA. Aging of skin. In: Wolff K, Goldsmith L, Gilchrest B, Paller A, Leffell D, Katz S, et al., editors. Fitzpatrick's dermatology in general medicine. 7th ed. New York: McGraw-Hill; 2008. p. 963-72.

3. World Health Organization. Pathology and genetic of skin tumors: melanocytic tumors. Lyon: International Agency for Research on Cancer (IARC); 2006. p. 51-77.

4. Markovic SN, Erickson LA, Rao RD, Weening $\mathrm{RH}$, Pockaj BA, Bardia A, et al. Malignant melanoma in the 21st century, part 1: epidemiology, risk factors, screening, prevention, and diagnosis. Mayo Clin Proc. 2007;82(3):364-74.

5. Buchan J, Roberts DL. Pocket guide to malignant melanoma. Oxford, UK: Blackwell Science Ltd; 2008. p.1-5.

6. Markovic SN, Erickson LA, Rao RD, Weening RH, Pockaj BA, Bardia A, et al. Malignant melanoma in the 21st century, part 2: staging, prognosis, and treatment. Mayo Clin Proc. 2007;82(4):490-513.

7. Homsi J, Kashani-Sabet M, Messina JL, Daud A. Cutaneous melanoma: prognostic factors. Cancer Control. 2005;12(4):223-9.

8. Kabigting FD, Nelson FP, Kauffman CL, Popoveniuc G, Dasanu CA, Alexandrescu DT. Malignant melanoma in African-Americans. Dermatol Online J. 2009;15(2):3.
9. Anger M, Friedhoper H, Fukutaki MF, Ferreira MC, Landman G. Primary cutaneous melanoma: an 18-year study. Clinics (Sao Paulo). 2010;65(3):257-63.

10. Pailoor J, Mun KS, Leow M. Cutaneous malignant melanoma: clinical and histopathological review of cases in a Malaysian tertiary referral centre. Malays J Pathol. 2012;34(2):97-101.

11. Káram-Orantes $\mathrm{M}$, Toussaint-Caire $\mathrm{S}$, Domínguez-CheritJ, Veja-Memije E. Clinical and histopathological characteristics of malignant melanoma cases seen at "Dr. Manuel Gea González" General Hospital. Gac Med Mex. 2008;144(3):19-23.

12. Roche LM,Xiao-ChengW, Vivien C, Elizabeth HB, Frank DG, Ahmedin J, et al. Cutaneous melanoma incidence and survival among Black, Asian, Pacific Islander and White populations in the United State. Clinical Medicine Insights. 2010;(3):15-24.

13. Takata M. Acral melanoma: clinical, biologic and molecular genetic characteristic. In: Murph M, editor. Melanoma in the clinicdiagnosis, management and complications of malignancy. Rijeka: InTech; 2011. p. 3-14.

14. Park HS, Cho KH. Acral lentiginous melanoma in situ: a diagnostic and management challenge. Cancers (Basel). 2010;(2):642-52.

15. Melanoma molecular map project. Melanoma: an introduction. [cited 2013 May 15] Available from: http://www. mmmp.org. 